\title{
Localized nonlinear waves in a semiconductor with charged dislocations
}

\author{
Vladimir I. Erofeev ${ }^{1 *}$, Anna V. Leonteva ${ }^{1}$, Alexey O. Malkhanov ${ }^{1}$ and Ashot V. Shekoyan ${ }^{2}$ \\ ${ }^{1}$ Mechanical Engineering Research Institute of Russian Academy of Sciences, 85, Belinskogo st., \\ Nizhny Novgorod, 603024, Russia \\ ${ }^{2}$ Institute of Mechanics of the National Academy of Sciences of the Republic of Armenia, 24B \\ Baghramyan Ave., Yerevan, 0019, Armenia
}

\begin{abstract}
To describe a nonlinear ultrasonic wave in a semiconductor with charged dislocations, an evolution equation is obtained that generalizes the well-known equations of wave dynamics: Burgers and Korteweg de Vries. By the method of truncated decompositions, an exact analytical solution of the evolution equation with a kink profile has been found. The kind of kink (increasing, decreasing) and its polarity depend on the values of the parameters and their signs. An ultrasonic wave in a semiconductor containing numerous charged dislocations is considered. It is assumed that there is a constant electric field that creates an electric current. The situation is similar to the case of the propagation of ultrasonic waves in piezoelectric semiconductors, but in the problem under consideration, instead of the electric field due to the piezoelectric properties of the medium, the electric field of dislocations appears.
\end{abstract}

\section{Mathematical model}

In the one-dimensional approximation, a nonlinear system of coupled equations for displacement $u$, dislocation movements $\xi$ and electric field $E$ have the following form [1]:

$$
\begin{gathered}
\rho \frac{\partial^{2} u}{\partial t^{2}}=c_{1} \frac{\partial^{2} u}{\partial x^{2}}+\beta b \frac{\partial \xi}{\partial x}+\frac{m}{2} \frac{\partial E}{\partial x}+\left(3 c_{1}+Q\right) \frac{\partial^{2} u}{\partial x^{2}} \frac{\partial u}{\partial x}+\frac{4}{3} q b^{2} \xi \frac{\partial \xi}{\partial x}+n E \frac{\partial E}{\partial x} \\
A \frac{\partial^{2} \xi}{\partial t^{2}}+B \frac{\partial \xi}{\partial t}=-\lambda \xi-\beta b \frac{\partial u}{\partial x}+\frac{P}{2} E-\frac{1}{2} \beta b\left(\frac{\partial u}{\partial x}\right)^{2}-\frac{4}{3} q b^{2} \xi \frac{\partial u}{\partial x}-\frac{d}{6} E^{a}-\frac{a}{3} E \xi \\
\frac{1}{2} \rho \frac{\partial \xi}{\partial x}+\varepsilon \frac{\partial E}{\partial x}+\frac{m}{2} \frac{\partial^{2} u}{\partial x^{2}}+\frac{d}{6} E \frac{\partial \xi}{\partial x}+\frac{q}{3} \xi \frac{\partial \xi}{\partial x}+2 n \frac{\partial E}{\partial x} \frac{\partial u}{\partial x}+2 n E \frac{\partial^{2} u}{\partial x^{2}}+\frac{m}{2} \frac{\partial^{2} u}{\partial x^{2}} \frac{\partial u}{\partial x} \\
=-n e .
\end{gathered}
$$

Here $\rho$ - density of the medium; $c_{1}, Q$ - linear and nonlinear elastic moduli; $A, B$ coefficients characterizing the mass and damping of dislocation oscillations; $\lambda$ - "stiffness" of dislocation; $\beta, q$ - coefficients of linear and nonlinear acousto-dislocation interaction; $b-$

\footnotetext{
* Corresponding author: erof.vi@yandex.ru
} 
component of the Burgers vector; $\varepsilon$-dielectric constant; $m, P, d, a, n$ - coefficients due to electrical, acoustic and dislocation interaction.

The equations for the components of the vector of electric current density have the following form:

$$
j_{i}=e \mu_{i k}\left(n_{0}+n(x)\right)\left[E_{k}^{0}+E_{k}(x)\right]+e N_{i k} \frac{\partial n}{\partial x_{i}},
$$

where $e$ - elemental charge, $\mu_{i k}$ - charge mobility tensor, $n_{0}$ - equilibrium charge concentration per unit volume of the medium, $n(x)$ - disturbance, $E_{0}$ - homogeneous direct current electric field, $E_{k}(x)$ - the electric field of a charged dislocation, and

$$
\vec{E}_{k}^{\prime}=\vec{E}_{0}+\vec{E}_{k}
$$

To these equations should be added the Maxwell equation

$$
\operatorname{rot} \vec{E}=0 \text {, }
$$

and equation

$$
-\frac{\partial n}{\partial t}+\operatorname{div} \vec{\jmath}=0
$$

In equations (1) - (3) we should change $\vec{E}$ to $\vec{E}_{k}^{\prime}$ with the help of formula (5), as well as $n^{\prime}=$ $n_{0}+n(x)$. Let us substitute (4) into equation (7), after we eliminate $j_{i}$, we get

$$
-\frac{\partial n}{\partial t}+\frac{\sigma}{e} \frac{\partial E}{\partial x}-v_{d} \frac{\partial n}{\partial x}+N \frac{\partial^{2} n}{\partial x^{2}}=\mu \frac{\partial}{\partial \tau}(n E),
$$

where $\sigma$ - electrical conductivity, $\mu$ - charge mobility, $v_{d}=-\mu E_{0}-$ charge drift velocity, $N$ - charge diffusion coefficient.

Let's move on to the new coordinate $\tau=\frac{x}{v}-t$, then the system of equations for semiconductors will take the following form:

$$
\begin{aligned}
\left(\rho-\frac{c_{1}}{v^{2}}\right) \frac{\partial^{2} u}{\partial \tau^{2}}= & -\frac{\beta b}{v} \frac{\partial \xi}{\partial \tau}+\frac{2 c_{1}}{v} \frac{\partial^{2} u}{\partial \tau \partial x}-\frac{P_{3}}{v} \frac{\partial E}{\partial \tau}-\frac{\left(3 c_{1}+Q\right)}{v^{3}} \frac{\partial^{2} u}{\partial \tau^{2}} \frac{\partial u}{\partial \tau}-\frac{4 q b^{2}}{3 v} \xi \frac{\partial \xi}{\partial \tau} \\
& -\frac{M}{v} E \frac{\partial E}{\partial \tau} \\
A \frac{\partial^{2} \xi}{\partial \tau^{2}}-B \frac{\partial \xi}{\partial \tau}= & -\Lambda_{0} \xi+\frac{b \beta}{v} \frac{\partial u}{\partial \tau}+P_{1} E-\frac{b \beta}{2 v^{2}}\left(\frac{\partial u}{\partial \tau}\right)^{2}-\frac{\gamma}{2} \xi^{2}+\frac{4}{3} \frac{q b^{2}}{v} \xi \frac{\partial u}{\partial \tau}-\frac{d}{6} E^{2} \\
& -\frac{a}{3} E \xi \\
-\frac{P_{2}}{2 v} \frac{\partial \xi}{\partial \tau}-\frac{\varepsilon}{v} \frac{\partial E}{\partial \tau}+ & \frac{P_{1}}{v^{2}} \frac{\partial^{2} u}{\partial \tau^{2}}-\frac{d}{6 v} E \frac{\partial \xi}{\partial \tau}-\frac{d}{2 v} \xi \frac{\partial E}{\partial \tau}-\frac{a}{3 v} \xi \frac{\partial \xi}{\partial \tau}+\frac{2 M}{v} E \frac{\partial^{2} u}{\partial \tau^{2}} \\
& +\frac{2 M}{v^{2}} \frac{\partial E}{\partial \tau} \frac{\partial u}{\partial \tau}-\frac{m}{v^{3}} \frac{\partial^{2} u}{\partial \tau^{2}} \frac{\partial u}{\partial \tau}=-e n,
\end{aligned}
$$




$$
\frac{\partial n}{\partial \tau}-\frac{\sigma}{e v} \frac{\partial E}{\partial \tau}+\frac{v_{d}}{v} \frac{\partial n}{\partial \tau}+\frac{N}{v^{2}} \frac{\partial^{2} n}{\partial \tau^{2}}=\frac{\mu}{v} \frac{\partial}{\partial \tau}(n E)
$$

where

$$
P_{3}=\frac{m}{2}+M E_{0}, \quad \Lambda_{0}=\frac{q}{3}+\lambda, \quad P_{1}=-\frac{d}{3} E_{c}-\frac{p}{2}, P_{2}=p+\frac{d}{3} E_{0}
$$

\section{Evolutionary Equation}

The system of nonlinear equations (9) - (12) will be studied by the method of evolution equation. The essence of the method is to derive one equation from the indicated system that preserves the basic physical properties of the original system, but is simpler for analysis. To do this, enter the small parameter $\varepsilon_{-} 1$ and give the values the corresponding order. We assume that $u \sim \varepsilon_{1}^{2}, \tau \sim \varepsilon_{1}, N \sim \varepsilon_{1}^{9}, A \sim \varepsilon_{1}^{3}, B \sim \varepsilon_{1}^{2}$. The order of the remaining quantities follows from equations (9) - (12). Determining the orders of magnitude for the largest quantities, we obtain the first system of equations, which is called the main one. Of the values of smaller orders, another system of equations is composed, from which we derive the evolutionary equation, sequentially excluding all quantities from the obtained new system, we obtain one equation for the quantity $u$. With exception, they use the main equations. This method is described in detail in books [2].

The system of main equations has the following form:

$$
\begin{gathered}
\left(\rho-\frac{c_{1}}{v^{2}}\right) \frac{\partial u}{\partial \tau}=-\frac{\beta b}{v} \xi-\frac{P_{3}}{v} E \\
-\Lambda_{0} \xi+\frac{b \beta}{v} \frac{\partial u}{\partial \tau}+P_{1} E=0 \\
-\frac{P_{2}}{2 v} \frac{\partial \xi}{\partial \tau}-\frac{\varepsilon}{v} \frac{\partial E}{\partial \tau}+\frac{P_{1}}{v^{2}} \frac{\partial^{2} u}{\partial \tau^{2}}=0 \\
\left(1+\frac{v_{d}}{v}\right) \frac{\partial n}{\partial \tau}-\frac{\sigma}{e} \frac{\partial E}{\partial \tau}=0
\end{gathered}
$$

The remaining equations have the form

$$
\begin{gathered}
\frac{2 c}{v} \frac{\partial^{2} u}{\partial \tau \partial x}-\frac{(3 c+Q)}{v^{3}} \frac{\partial^{2} u}{\partial \tau^{2}} \frac{\partial u}{\partial \tau}-\frac{4 q b^{2}}{3 v} \xi \frac{\partial \xi}{\partial \tau}-\frac{M}{v} E \frac{\partial E}{\partial \tau}=0 \\
A \frac{\partial^{2} \xi}{\partial \tau^{2}}-B \frac{\partial \xi}{\partial \tau}=-\frac{b \beta}{2 v^{2}}\left(\frac{\partial u}{\partial \tau}\right)^{2}-\frac{\gamma}{2} \xi^{2}+\frac{4}{3} \frac{q b^{2}}{v} \xi \frac{\partial u}{\partial \tau}-\frac{d}{6} E^{2}-\frac{a}{3} E \xi=0 \\
-\frac{d}{6 v} E \frac{\partial \xi}{\partial \tau}-\frac{d}{2 v} \xi \frac{\partial E}{\partial \tau}-\frac{a}{3 v} \xi \frac{\partial \xi}{\partial \tau}+\frac{2 M}{v} E \frac{\partial^{2} u}{\partial \tau^{2}}+\frac{2 M}{v^{2}} \frac{\partial E}{\partial \tau} \frac{\partial u}{\partial \tau}-\frac{m}{v^{3}} \frac{\partial^{2} u}{\partial \tau^{2}} \frac{\partial u}{\partial \tau}=-e n \\
\frac{N}{v^{2}} \frac{\partial^{2} n}{\partial \tau^{2}}=\frac{\mu}{v} \frac{\partial}{\partial \tau}(n E)
\end{gathered}
$$

From the system of equations (17) - (20) the evolutionary equation will be derived. But first, from the system of principal equations, it is necessary to derive the relations among the quantities $\xi, E, n$ and displacement $u$. In equations (17) - (20) all quantities are sequentially 
eliminated, then one equation for the function $u$ is obtained. When performing this procedure in nonlinear terms, where presented the quantities $\xi, E$ and $\mathrm{n}$, as well as in the terms $\frac{\partial^{2} \xi}{\partial \tau^{2}}, \frac{\partial \xi}{\partial \tau}$, the elimination is made by the main members. As a result, for the function $u$ we obtain the following evolutionary equation:

$$
-\frac{\partial \psi}{\partial x}+q_{1} \frac{\partial^{3} \psi}{\partial \tau^{3}}+q_{2} \frac{\partial^{2} \psi}{\partial \tau^{2}}+q_{3} \frac{\partial}{\partial \tau}\left(\psi \frac{\partial \psi}{\partial \tau}\right)+q_{4} 2 \psi \frac{\partial \psi}{\partial \tau}=0
$$

where

$$
\begin{gathered}
\psi=\frac{\partial u}{\partial \tau}, q_{1}=-\frac{A T_{2} \beta b}{2 v \Lambda_{0} c}, v_{0}^{2}=\frac{c}{\rho}-\frac{b^{2} \beta^{2}}{\rho \Lambda_{0}}, \\
q_{2}=-\frac{1}{2 v c}\left\{\beta b T_{3}+\frac{v+v_{d}}{2 v \sigma}\left[\frac{P_{2} P_{3} T_{2}^{2}}{2}-\frac{P_{3}}{v}\left(\mathrm{P}_{1}-\varepsilon T_{1}-\frac{N \sigma T_{1} v}{v+v_{d}}\right)\right]\right\}, \\
q_{3}=-\frac{v+v_{d}}{2 \sigma v^{4} c}\left(2 M T_{1}-\frac{d T_{1} T_{2}}{3}-m-\frac{a T_{2}^{2}}{3}\right)\left(\frac{\beta b P_{1}}{\Lambda_{0}}+P_{3}\right), \\
q_{4}=\frac{1}{2 v^{2} c}\left[T_{4} \beta b+\frac{2 M T_{1}\left(v+v_{d}\right) P_{3}}{\sigma v^{2}}+3 c+Q+\frac{4 q b^{2} T_{2}^{2}}{3}+T_{1}^{2} M\right], \\
T_{3}=\frac{1}{\Lambda_{0}}\left\{B T_{2}+\frac{P_{1} P_{2} T_{2}\left(v+v_{d}\right)}{2 v^{2} \sigma}-\frac{v+v_{d}}{v^{2} \sigma}\left[\mathrm{P}_{1}-\varepsilon T_{1}-\frac{N \sigma T_{1} v}{\left(v+\nu_{d}\right)^{2}}\right]\right\}, \\
T_{4}=\frac{1}{v^{2}}\left(\frac{2 M T_{1} P_{1}}{v^{2} \sigma}-\frac{b \beta}{2}-\frac{\gamma T_{2}^{2}}{2}+4 q b^{2} T_{2}-\frac{T_{1} d}{6}-\frac{a T_{1} T_{2}}{3}\right) .
\end{gathered}
$$

\section{Solution}

With the help of traveling wave variables $\psi(x, \tau)=\psi(z), z=\frac{x}{v}-\tau$, we find the solution of the equation by the truncated expansion method $[3,4]$ in the form:

$$
\psi(z)=-\frac{q_{1}}{q_{3}}\left|\frac{2 q_{1} q_{4}-q_{2} q_{3}}{q_{1} q_{3}}\right| \operatorname{th}\left(\left|\frac{2 q_{1} q_{4}-q_{2} q_{3}}{2 q_{1} q_{3}}\right| z\right)+\frac{2 q_{1} q_{4}-q_{2} q_{3}}{q_{3}^{2}},
$$

where $v$ - the velocity of a nonlinear wave is determined by the expression

$$
v=-\frac{q_{3}^{2}}{2 q_{4}\left(2 q_{1} q_{4}-q_{2} q_{3}\right)} .
$$

The solution has a kink profile. The kind of kink (increasing, decreasing) and its polarity depend on the values of the parameters and their signs. In the case when all the coefficients of the equation are positive, we have a decreasing kink in the upper half-plane. 


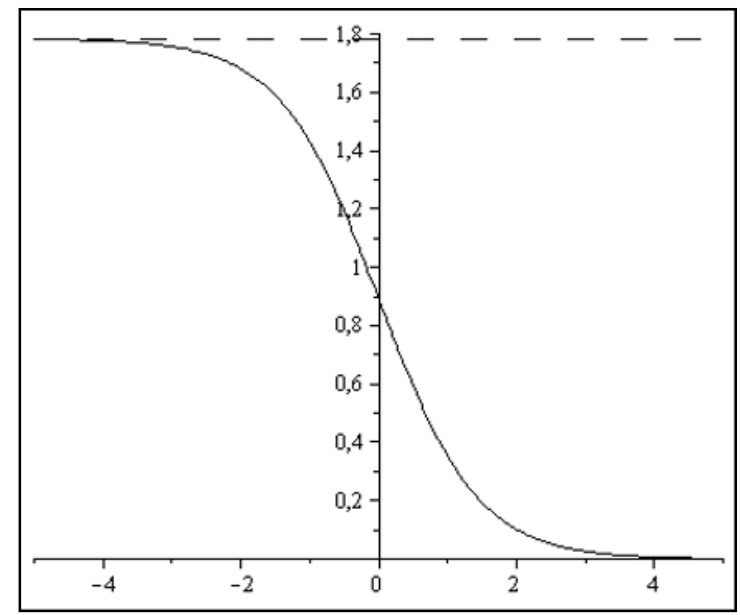

Fig. 1 The kink profile

In equation (21), the term with the coefficient $q_{1}$ is prescribed by dispersion. When we substitute $q_{1}=q_{3}=0$ from (21) we get an equation of Burgers type. The term with the coefficient $q_{2}$ is prescribed by dissipation. When $q_{2}=q_{3}=0$ from (21) we obtain the Korteweg - de Vries equation.

In conclusion, we note that the study of linear and nonlinear ultrasonic waves in dielectric crystals with charged dislocations was carried out earlier in [5].

\section{Acknowledgements}

The work was carried out within the Russian state task for fundamental scientific research for 2021-2023 (the topic No. 0030-2021-0025) and supported by RFBR (projects 20-3870158, 18-29-10073).

\section{References}

1. Erofeev V.I., Leontieva A.V., Shekoyan A.V. Nonlinear waves in a semiconductor with charged dislocation. Topical Problems of Continuum Mechanics. Proceeding of VI International Conference. 01- 06 October, Dilijan, Armenia. Yerevan: National Academy of Sciences of Armenia. Institute of Mechanics. p.156-159. (2019)

2. Bagdoev A.G., Erofeyev V.I., Shekoyan A.V. Wave Dynamics of Generalized Continua. Berlin, Heidelberg: Springer-Verlag, 274 p. (2016)

3. Kudryashov N.A. Analitical Theory of Nonlinear Differential Equations. Ins. Comp'"ut. Issled., Moscow-Izhevsk, 360 p. (2004) (in Russian).

4. Kudryashov N.A. Nonlinear Mathematical Physics Techniques. Intellekt, Dolgoprudny, 368 p. (in Russian). (2010)

5. Shekoyan A.V. Linear and nonlinear waves in crystals with charged dislocations. Physics of the Solid State. Vol.54, No 4, p.816-819. (2012) 